\title{
Brouwer 不动点定理与多态的稳定性
}

\author{
陆 寿 坤 \\ (杭州大学数学系)
}

Brouwer 不动点定理是数学上一个重要定理, 它告诉我们: $n$ 维实心球 $V^{n}$ 的任意一个连 续自映射 $\varphi: V^{n} \rightarrow V^{n}$ 至少有一个不动点, 即至少有一个点 $x_{0} \in V^{n}$, 使得 $\varphi\left(x_{0}\right)=x_{0}{ }^{[1]}$. 这 里, $n$ 维实心球 $V^{n}$ 改为 $n$ 维单形 $\underline{S}^{n}$, 定理仍成立.

Brouwer 不动点定理在数学上有着广泛的应用, 而本文的目的是给出这个定理在研究生物 多态现象的稳定性方面的重要应用.

多态现象是生物领域中一种相当广泛的现象: 例如, 某个人类群体, 按其血型, 刚好区分 为 $A$ 型、 $B$ 型、 $A B$ 型、 $O$ 型. 则此四种血型就构成这个人类群体的血型多态现象 (四态现象). 这是一个表现型多态现象的例子. 其实, 在生物领域中表现型多态现象多至无穷. 此外, 还有 举不胜举的基因多态现象与基因型多态现象: 如, 群体在一个位点上, 若有 $A$ 与 $a$ 二个等位 基因, 则此二个等位基因就构成该群体 (在上述位点上)的基因多态现象 (二态). 若存在复等 位基因: $a_{1}, a_{2}, \cdots, a_{n}$, 则此 $n$ 个复等位基因就构成上述群体的基因多态现象 ( $n$ 态). 一个 群体有时还会出现染色体多态现象; 如在果蝇的许多物种中由于某些个体出现染色体畸变而 引起这些物种的染色体多态现象. 特别是把任何一个群落看成一个多态现象, 只要把这群落 中每个物种看成一个态, 那么这群落中所有物种就构成一个多态现象.

研究多态现象的稳定性在遗传、进化论、生态学与经济理论方面都有重要意义. 然而, 在 生物界至今还没有人对多态现象下过确切定义, 虽然象福特等人为多态现象下过定义 ${ }^{[2]}$, 但这 些定义不确切. 而本文中所指的多态现象是如下的.

定义 1 一个对象(即一个群体或一个群落)的全体成员如果按照某种特征 (如某性状、 某位点上基因或基因型、某种染色体以及按照物种等)区分为 $\boldsymbol{n}$ 种不同类型,则称此 $\boldsymbol{n}$ 种类型 为该对象的上述特征的 $n$ 态现象 (当 $n \geqslant 2$ 时, 也称多态现象). 其中每个类型称为一个态.

如果, 用 $G_{1}, G_{2}, \cdots, G_{n}$ 分别表示上述 $n$ 个态; 且用 $\lambda_{1}, \lambda_{2}, \cdots, \lambda_{n}$ 表示它们的频率, 那 么,我们引进

定义 2 表达式:

$$
\lambda_{1} G_{1}+\lambda_{2} G_{2}+\cdots+\lambda_{n} G_{n},
$$

其中 $\lambda_{i} \geqslant 0, i=1,2, \cdots, n$; 且 $\sum_{i=1}^{n} \lambda_{i}=1$, 称为上述对象的多态结构表达式. (*)式 含意是上述对象是由 $n$ 个态: $G_{1}, G_{2}, \cdots, G_{n}$ 组成, 每态前面的系数 $\lambda$ 表示该态成员数在群 体中所占比例(即频率).

多态结构表达式 (下文简称 $P-S$ 式) 不仅指出对象中具有那些态, 而且还指明了它们的频

本文 1981 年 9 月 14 日收到. 
率为多大. 这样, 用 $P-S$ 式来描述多态现象就比较明确. 其实, 生物领域中任何多态现象(除 那些具有无穷多态的现象之外) 都可以用相应的 $P-S$ 式明确地表示. 特别当对象是一个孟德 尔群体, 且把 (*)式中的 $G_{1}, G_{2}, \cdots, G_{n}$ 理解为该群体(在一个位点上)的全体基因型时, 那 么, 这时的基因型 $P-S$ 式实际上就是基因型展开式. 因此, $P-S$ 式是群体遗传结构的基因型 展开式的这一概念的推广.

然而, 当对象是一个群落, 那么这个群落的 $P-S$ 式能够反映该群落的结构. 如设有一个群 落的 $P-S$ 式为:

$$
\frac{1}{6} G_{1}+\frac{2}{6} G_{2}+\frac{1}{6} G_{3}+\frac{2}{6} G_{4},
$$

其中 $G_{1}$ 表示猫、 $G_{2}$ 表示老鼠、 $G_{3}$ 表示土蜂、 $G_{4}$ 表示红三叶草. 这个 $P-S$ 式明确地指出该群 落是由猫、老鼠、土蜂、红三叶草组成, 且其比例分别占总数的 $1 / 6 、 2 / 6 、 1 / 6$ 和 $2 / 6$. 所以, 从 群落 $P-S$ 式看群落的结构就一目了然.

通常，对象的多态现象可能出现如下二种类型变化：第一种是多态现象在变化过程中出 现新的态, 如由于基因突变产生新的基因(即新的态), 又如从别处迁人一些新的态等等; 第二 种是多态现象在变化过程中无新态出现, 仅仅是原有的态的频率在变化 (包括某些频率变为 0 , 或某些频率由非 0 变成 0 再变成非 0 的情形等等). 如当群体不发生基因突变, 没有新基 因迁人, 仅仅是选择、迁出、交配方式以及群体含量等因素在起作用时, 那么该群体的基因多态 现象与基因型多态现象是属于第二种变化. 同理, 当一个群落无新物种迁入时, 通常它的多态 现象的变化,仅仅是这些物种的频率在变化.

下文,我们仅讨论属于第二种类型的变化. 为此,先看 $P-S$ 式的几何上的解释.

在平面上, 任取一个一维单形 $\underline{S}^{1}=\left(a_{0}^{0} a^{1}\right)$ (即 $\underline{S}^{1}$ 是闭线段)(图 1). 则 $\underline{S}^{1}$ 上任一点 $x_{0}$ 可表成

$$
x_{0}=\mu_{0} a^{0}+\mu_{1} a^{1} \text {, }
$$

其中 $\mu_{0} \geqslant 0, \mu_{1} \geqslant 0$, 且 $\mu_{0}+\mu_{1}=1$.

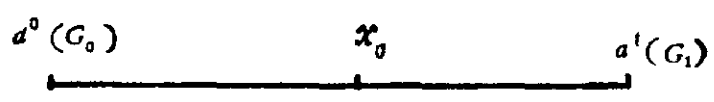

图 1

另外, 若有一对象仅有二态 $G_{0}, G_{1}$, 且它们的频率值恰好是上面的 $\mu_{0}, \mu_{1}$. 于是这对象的 二态结构表达式为

$$
\mu_{0} G_{0}+\mu_{1} G_{1} \text {, }
$$

其中 $\mu_{0} \geqslant 0, \mu_{1} \geqslant 0$ 且 $\mu_{0}+\mu_{1}=1$. 比较 (1) 与 ( $\left.1^{\prime}\right)$ 式, 它们十分相似. 如果让顶点 $a_{0}$ 表示态 $G_{0}, a^{1}$ 表示 $G_{1}$. 那么 (1) 式中点 $x_{0}$ 就表示上面的二态结构表达式 (1'). 这样, 单形 $\underline{S}^{1}=\left(a^{0}, a^{1}\right)$ 中的点与集合: $\left\{\mu_{0} G_{0}+\mu_{1} G_{1} \mid\right.$ 其中, $\mu_{0} \geqslant 0, \mu_{1} \geqslant 0$, 且 $\left.\mu_{0}+\mu_{1}=1\right\}$ 中元 素之间可以建立一一对应*. 其中向量 $\left(\mu_{0}, \mu_{1}\right)$ 的二个分量既可以作为单形 $\underline{S}^{1}$ 中的点 $x_{0}$ 的坐标, 也可以作为式 $\left(1^{\prime}\right)$ 中二态 $G_{0}, G_{1}$ 的频率. 整个单形 $\underline{S}^{1}=\left(a^{0}, a^{1}\right)$ 表示整个集合: $\left\{\mu_{0} G_{0}+\mu_{1} G_{1} \mid \mu_{0} \geqslant 0, \mu_{1} \geqslant 0\right.$, 且 $\left.\mu_{0}+\mu_{1}=1\right\}$, 而后者表示具有态 $G_{0}$ 与 $G_{1}$ 的一切多态现 象 (这里包括仅有一态 $G_{0}$ 或 $G_{1}$ 的情形).

* 当频率为一个无理数 $r \in(0,1)$ 时, 则把 $r$ 看成 (一个有实际意义的)以有理数为项 $a_{n} \in[0,1]$ 的频率序列 $\left\{a_{n}\right\}$ 的极限状态, 即 $r=\lim _{m-\infty} a_{n}$. 
同理,对于三态结构表达式: $\mu_{0} G_{0}+\mu_{1} G_{1}+\mu_{2} G_{2}$ 可以在平面上取一个三角形(即二维单 形 $\left.\underline{S}^{2}=\left(a^{0}, a^{1}, a^{2}\right)\right)($ 图 2) 进行类似讨论.

一般地, 在 $n$ 维欧氏空间中, 任取 $n+1$ 个占有 最广位置点组 ${ }^{[1]}: a^{0}, a^{1}, \cdots, a^{n}$, 作一个 $n$ 维单形 $\underline{S}^{n}=\left(a^{0}, a^{1}, \cdots, a^{n}\right)$, 则 $\underline{S}^{n}$ 上任一点 $u$ 可表成

$$
u=\lambda_{0} a^{0}+\lambda_{1} a^{1}+\cdots+\lambda_{n} a^{n},
$$

其中, $\lambda_{i} \geqslant 0, i=0,1, \cdots, n$; 且 $\sum_{i=0}^{n} \lambda_{i}=1$.

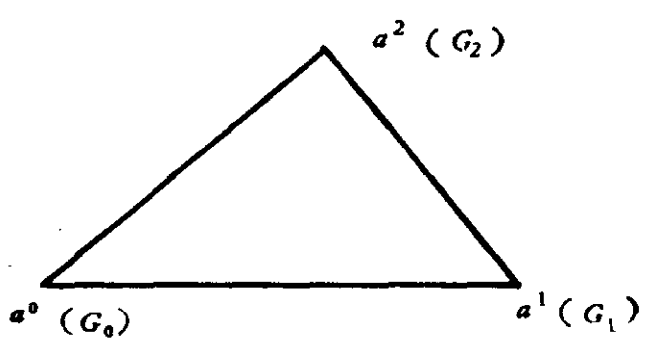

图 2

另外, 若有一个对象 (记作 $A$ ) 具有 $n+1$ 个态: $G_{0}, G_{1}, \cdots, G_{n}$, 且它们的频率值恰 好是上面的 $\lambda_{0}, \lambda_{1}, \cdots, \lambda_{n}$, 则对象 $A$ 的 $P-S$ 式为

$$
\lambda_{0} G_{0}+\lambda_{1} G_{1}+\cdots+\lambda_{n} G_{n},
$$

其中 $\lambda_{i} \geqslant 0, i=0,1, \cdots, n$; 且 $\sum_{i=0}^{n} \lambda_{i}=1$.

如果让顶点 $a^{i}$ 表示态 $G_{i}(i=0,1, \cdots, n)$, 则 $S^{n}$ 中点与集合: $\left\{\lambda_{0} G_{0}+\lambda_{1} G_{1}+\cdots+\right.$ $\lambda_{n} G_{n} \mid$ 其中, $\lambda_{i} \geqslant 0, i=0,1, \cdots, n$; 且 $\left.\sum_{i=0}^{n} \lambda_{i}=1\right\}$ 中元素可以建立一一对应. 这里, 点 $u$ 表示 $P-S$ 式 (2). 整个 $n$ 维单形 $S^{n}$ 就表示具有态: $G_{0}, G_{1}, \cdots, G_{n}$ 的一切多态现象（包括 $\left\{G_{i}, i=0,1, \cdots, n\right\}$ 中某些态不出现情形).

因此, 研究多态现象的第二类变化问题就归结为研究 $n$ 维单形 $\underline{S}^{n}$ 到它自身的变化问题. 特别, 当我们把 Brouwer 不动点定理用上面的多态现象的语言进行意译的话, 就得到如下的重 要.

定理 设有一个多态(具态: $G_{0}, G_{1}, \cdots, G_{n}$ ) 现象在变化过程中仅仅是这些态的频率 在变化, 且设变化规律 $\boldsymbol{\varphi}$ 是频率向量 $\left(\lambda_{0}, \lambda_{1}, \cdots, \lambda_{n}\right)$ 的一个连续映射*, 那么定存在一个特 定的 $P-S$ 式: $\bar{\lambda}_{0} G_{0}+\bar{\lambda}_{1} G_{1}+\cdots+\bar{\lambda}_{n} G_{n}$, 使得多态现象在上述变化过程中, 此式不变（即 当对象处于 $\bar{\lambda}_{0} G_{0}+\bar{\lambda}_{1} G_{1}+\cdots+\bar{\lambda}_{n} G_{n}$ 时,在上述变化过程中是稳定的).

作为一个应用, 有如下例子:

设有态 $G_{0}, G_{1}, \cdots, G_{n}$ 的一个孟德尔群体,在世代相传过程中, 受到如下一种选择力的 作用: 当 $\left\{G_{i}\right\}$ 中间的某些态的频率变成很小时, 选择对这些态有利, 而当它们的频率变成很 大时, 选择对这些态反而不利, 且设这种选择力是频率向量的连续映射时, 则定有一个 $P-S$ 式: $\bar{\lambda}_{0} G_{0}+\bar{\lambda}_{1} G_{1}+\cdots+\bar{\lambda}_{n} G_{n}$ 存在, 使得它在世代相传过程中不变. 即这时态不变, 频率也不 变.

\section{参考文献}

[1] 江泽涵,拓扑学引论, 上海科技出版社, 1978 .

[2] l. M. 薛柏德,自然选择和遗传,第四章,科学出版社, 1966 .

* 这里的 $\varphi$ 是欧氏空间的子空间: $\left\{\left(\lambda_{0}, \lambda_{1}, \cdots, \lambda_{n}\right) \mid\right.$ 其中 $\left.\sum_{j=0}^{n} \lambda_{i}=1, \lambda_{i} \geqslant 0, i=0,1, \cdots, n\right\}$ 到它自身的一 个连续映射. 